\title{
A Kind of Reducing PAPR Algorithm Based on OFDM System Model in Wireless Sensor Network
}

\author{
Chen Chun, Zhang Fan, Ma Pengfei, Yang Haibo, Zhang Jichun, Wei Wenbo, Ni Min \\ New Star Institute of Applied Technology Hefei, Anhui Province, China \\ salas0527@163.com
}

\begin{abstract}
In Orthogonal Frequency Division Multiplexing (OFDM) used in Wireless Sensor Network (WSN), in order to restrain the intermodulation interference between subcarrier and outof-band radiation brought by system nonlinearity of Peak-to-Average Ratio (PAPR), a kind of joint algorithm is put forward, which is based on Selective Mapping (SLM) and Repeated Clipping and Filtering (RCF). By theoretical analysis, the joint scheme applied in OFDM system can reduce the PAPR dramatically. Further, simulation result afterwards verifies the effectiveness of the method, which can avoid Bit Error Rate (BER) performance degradation. So the method proposed in the paper proves to be very effective.

Index Terms - Orthogonal Frequency Division Multiplexing (OFDM), Peak-to-Average Ratio (PAPR), Selective Mapping (SLM), Repeated Clipping and Filtering (RCF), Bit Error Rate (BER)
\end{abstract}

\section{Introduction}

In Wireless Sensor Network (WSN), the node status is very important, which has the function of end node and routing. On the one hand, it implements for data acquisition and processing. On the other hand, it implements for data fusion and routing. So the appropriate signal modulation mode of the node plays an important role in the efficiency, robustness, and life cycle of the whole system.

The nodes has own specific communication structure, which can adopt some modulation modes. Among these, Orthogonal Frequency Division Multiplexing (OFDM) is a special kind of multi-carrier transmission technology, which has tremendous benefits. So it is gradually being used in the nodes of WSN [1]. However, OFDM has a high Peak-toAverage Ratio (PAPR), which can produce intermodulation interference between subcarrier and out-of-band radiation. In order to control the disadvantage influence, some decisive measurement must be used to control the PAPR of OFDM. And it is main task of the paper.

\section{Node Structure and Modulation Mode Choice of WSN}

A. Node Structure of WSN

The basic composition of node in WSN includes the following three basic units: Sensor Unit (including sensor and D/A conversion function module), Processing Unit (including processor, memory), Communication Unit (including wireless communication module). In addition, the other choosable functional units includes: Node Positioning System, Mobile System, as well as Self-powered System, etc. So the node structure of WSN is shown in Fig. 1.

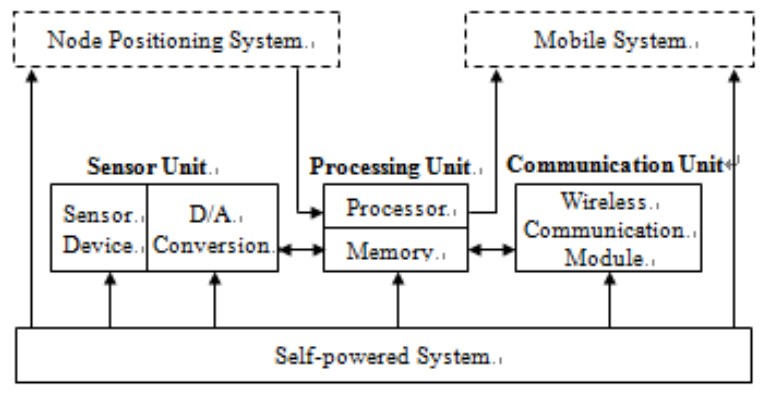

Fig. 1 Node Structure of WSN

B. Modulation Mode Choice of WSN

At present, the optional modulation mode includes: ASK, FSK, PSK, QAM, OFDM, etc.

Among these, ASK, FSK and PSK, are used relatively more often in WSN. With the rapid development of digital circuit, OFDM has gradually been used either.

OFDM is a special kind of multi-carrier transmission technology, which can effectively combat multipath fading, overcome the Symbol Interference (SI). And it can realize high speed data transmission with high spectrum utilization, and can significantly reduce the computation complexity, which has been widely used in Digital Auto Broadcast (DAB-T), Digital Video broadcasting (DVB-T), Wireless Local Area Networks (WLANs) (IEEE 802.11a, ETSI Hiperlan2) and Wireless Metropolitan Area Networks (IEEE 802.16d) [2], and other technical fields. In addition, in the 4th generation mobile communication system, OFDM is chosen as the core transmission technology. For example, the goal of B3G/4G proposed by the International Telecommunications Union (ITU) is to support downlink data transmission rate up to $100 \mathrm{Mb} / \mathrm{s}$ in the high-speed mobile environment, and to support downlink data transmission rate up to $1 \mathrm{~Gb} / \mathrm{s}$ in the static and interior environment. However, one of the main defect of OFDM signal is that its PAPR is high, which limits the extensive using of OFDM system. When the input and output data is in same-phase, the output signal of OFDM will be of great peak value. Although emerging probability of high PAPR is very low, in order to transfer these OFDM signals without distortion, the linearity of High Power Amplifier (HPA) and A/D converter is required very high. If nonlinearity appears when in high peak value power, it can produce intermodulation interference between subcarrier and out-ofband radiation, and can reduce the performance of OFDM system dramatically. So it is very important for OFDM system 
to use appropriate technology to reduce the PAPR. In the paper, a kind of joint algorithm is put forward, which is based on Selective Mapping (SLM) and Repeated Clipping and Filtering $(\mathrm{RCF})$. And it tries to reduce the PAPR when the OFDM model is adopted by the nodes in WSN, which can effectively avoid the performance degradation of Bit Error Rate (BER) of information transmitted.

\section{The Definition and Measurement of PAPR in OFDM}

In an OFDM system, the binary data stream is mapped with M-ary PSK or 16-QAM modulation. And then the serial data sequence is converted to a parallel format. After applied Inverse Fast Fourier Transform (IFFT) and added cyclic prefixes to $\boldsymbol{N}$ parallel sub symbols, an OFDM symbol can be attained. And in the time domain it can be described as follows.

$$
x_{n}=\frac{1}{\sqrt{N}} \sum_{k=0}^{N-1} X_{k} \cdot e^{\frac{j 2 \pi k n}{N}}, \quad n=0,1, \cdots, N-1 .
$$

In the equation above, $\boldsymbol{X}_{\boldsymbol{k}}$ is the modulated symbol, $\boldsymbol{n}$ stands for a discrete time index, and $N$ means the number of the subcarriers.

The PAPR, defined as the ratio of the peak to the average power of the OFDM signal, can be expressed as

$$
P A P R=\frac{\max \left|x_{n}\right|^{2}}{E\left[\left|x_{n}\right|^{2}\right]}
$$

In Formula (2), $\boldsymbol{E}[\mathrm{]}$ stands for the expectation value.

Because the peak value of the signal power doesn't often appear, so only researching the instantaneous PAPR is not significant. In practice, the Complementary Cumulative Density Function (CCDF) of the PAPR is frequently used to measure the performance of PAPR reduction, which means the probability that the PAPR is more than certain threshold value. The CCDF of the PAPR of an OFDM symbol with Nyquist sampling rate can be derived as follows.

$$
P(P A P R>z)=1-\left(1-e^{-z}\right)^{N}
$$

\section{IV . The Proposed Method}

In this paper, simplified SLM [3] and RCF algorithm are adopted together to reduce the PAPR. The reason for choosing the two algorithms is listed as follows.

Firstly, SLM algorithm has good PAPR reduction performance, which doesn't bring about distortion to the transmitted signal, and is simple in theory. Besides these advantages above, simplified SLM algorithm can still decrease the number of the SI effectively. Therefore, it can make the best of the frequency spectrum resource. Secondly, RCF algorithm is well known because of its prominent PAPR reduction performance and reduction for the overall peak regeneration. So, we try to combine the advantage of above two methods, which may offer a good way to reduce the PAPR in OFDM.

\section{A. The Simplified SLM Scheme}

The block diagram of simplified SLM scheme is shown in Fig. 2.

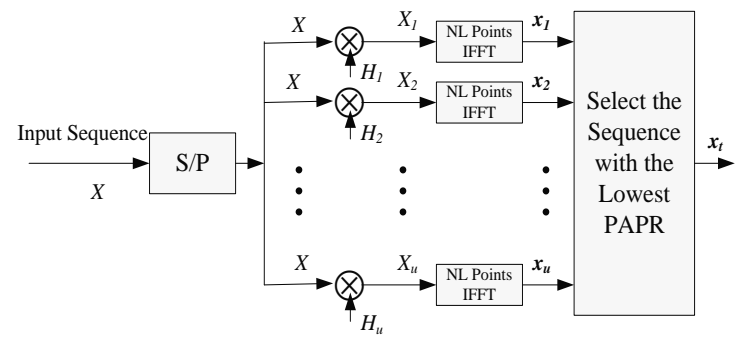

Fig. 2 Block Diagram of Simplified SLM Scheme

The number $N$ of the subcarriers also denotes the rank of the Hadamard matrix $\operatorname{hadamard}(\boldsymbol{N})$. With regard to the $\boldsymbol{u}$ scrambling sequences, only the first $\boldsymbol{u}$ columns of $\operatorname{hadamard}(\boldsymbol{N})$ is needed. For example, when the number $\mathrm{N}$ of the subcarriers is 128 , the Hadamard matrix is as follows.

$$
H_{128}=\text { hadamard }(128)=\left[\begin{array}{rrrlc}
1 & 1 & \cdots \cdots & 1 & 1 \\
1 & -1 & \cdots \cdots & 1 & -1 \\
1 & 1 & \cdots \ldots & -1 & -1 \\
\vdots & \vdots & \ddots & \vdots & \vdots \\
1 & -1 & \cdots \cdots & 1 & -1
\end{array}\right]
$$

In Fig. 2, $\boldsymbol{H}_{\boldsymbol{I}}$ stands for the first column of the Hadamard matrix $\boldsymbol{H}_{128}$, and so on. The letter $\boldsymbol{u}$ indicates that $\boldsymbol{u}$ phase adjustment sequences are used. $\boldsymbol{X}$ is the mapped sequence with some modulation scheme. The $I$ th scrambled vector $\boldsymbol{X}_{\boldsymbol{i}}=\boldsymbol{X}_{\boldsymbol{0}, \boldsymbol{i}}, \boldsymbol{X}_{1}, \boldsymbol{i}, \ldots, \boldsymbol{X}_{\boldsymbol{N}-\boldsymbol{1}, \boldsymbol{i}}(\mathbf{1} \leq \boldsymbol{i} \leq \boldsymbol{u})$ is transformed using an oversize IFFT. For an oversampling factor of $\boldsymbol{L}, \boldsymbol{X} \boldsymbol{i}$ is extended by adding $N(\boldsymbol{L}-1)$ zeros in the middle of the vector, which leads to trigonometric interpolation of the signal in time domain [4]. That means, the sequence $\boldsymbol{X}_{\boldsymbol{i}}$ becomes

$$
X_{i}=X_{0, i}, X_{1}, i, \ldots, X_{N / 2-1}, i, 0, \ldots, 0, X_{N / 2}, i, \ldots, X_{N-1}, i
$$

Then the IFFT is performed to the sequence $\boldsymbol{X}_{\boldsymbol{i}}(1 \leq \boldsymbol{i} \leq \boldsymbol{u})$, resulting in $\boldsymbol{x}_{\boldsymbol{i}}(1 \leq \boldsymbol{i} \leq \boldsymbol{u})$. After computing and comparing the PAPR of the sequence $\boldsymbol{x}_{\boldsymbol{i}}(1 \leq \boldsymbol{i} \leq \boldsymbol{u})$, the sequence with the lowest PAPR, denoted as $\boldsymbol{x}_{\boldsymbol{t}}$, is selected to transmit.

In simplified SLM algorithm, the phase sequences are generated by Hadamard matrix. As the matrix is constant and available both at the transmitter and the receiver, there is no need to transmit the factors of the matrix as the SI, which greatly simplifies the search for optimal phase sequences and reduces the number of the SI.

\section{B. The RCF Algorithm}

In Fig. 3, the implementation process of RCF scheme is shown [5]. The absolute-magnitude of the input complex sequence $\boldsymbol{x}_{\boldsymbol{t}}$ from the SLM scheme is clipped. The clipping process can be expressed as

$$
y= \begin{cases}x, & x \square A \\ A, & x>A\end{cases}
$$




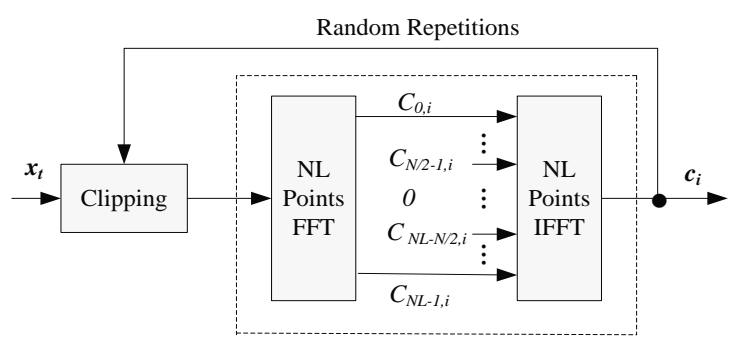

Frequency Domain Filtering

Fig. 3 Block Diagram of the RCF Algorithm

In formula (6), $\boldsymbol{x}$ means the absolute-magnitude of the signal that will be clipped, $\boldsymbol{y}$ is the output after the clipping process, and $\boldsymbol{A}$ is the clipping threshold value. In practice, the Clipping Ratio (CR) is used frequently to measure the clipping level in the form of $\mathrm{dB}$, which is defined as the ratio of the maximum power of the clipped signal to the average power of the unclipped signal. It can be expressed as

$$
C R=20 \log _{10} \frac{A}{\sigma} \quad(d B)
$$

In the equation above, $\sigma$ is the Root-Mean-Square (RMS) power of the unclipped signal. In addition, according to the need of the clipping effect, the clipping threshold value $\boldsymbol{A}$ could be chosen to be $60 \%$ or $50 \%$ of the peak value of the signal [6].

The clipping is followed by filtering to remove out-ofband noise. The filtering is implemented in the way of frequency domain filtering. That means, the FFT and IFFT should be executed at a time respectively. The FFT transforms the clipped signal back into the discrete frequency domain resulting in $\boldsymbol{C}_{\boldsymbol{k}, \boldsymbol{i}}$. The both ends of $\boldsymbol{C}_{\boldsymbol{k}, \boldsymbol{i}}$ are passed unchanged to the inputs of the IFFT while the components in out-of-band are nulled. So it is shown as follows.

$$
C_{k, i}= \begin{cases}c_{k, i}^{\prime}, & k=0,1, \cdots, \frac{N}{2}-1 \quad \text { and } k=N L-\frac{N}{2}, \cdots, N L-1 \\ 0, & k=\frac{N}{2}, \cdots, N L-\frac{N}{2}-1\end{cases}
$$

In the equation (8), $\boldsymbol{k}$ denotes the $\boldsymbol{k}$ th subcarrier. Then the IFFT operation converts the frequency domain signals $\boldsymbol{C}_{\boldsymbol{k}, \boldsymbol{i}}$ back to the time domain, which produces the filtered clipped signal $\boldsymbol{c}_{\boldsymbol{i}}$ at the output of the frequency domain filtering. For the better effects for PAPR reduction, the process of the clipping and filtering can be implemented for several times.

Compared to the ordinary clipping method, RCF algorithm can decrease the out-of-band noise immensely owing to the novel method of frequency domain filtering, which can also suppress the peak regeneration of the clipped signal and then can reduce the PAPR.

\section{The Joint Scheme Applied in OFDM System}

The basic principle of joint scheme applied in OFDM system is described in Fig. 4.

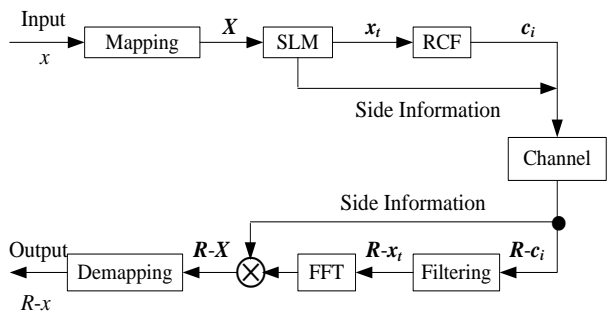

Fig. 4 Block Diagram of the Joint Scheme Applied in OFDM System

The input data stream $\boldsymbol{x}$ made of $2 \boldsymbol{N}$ binary numbers is first mapped in the form of Gray code with QPSK modulation in the paper. Hence the consequent sequence $\boldsymbol{X}$ becomes 128 complex signals composed of $1+\mathrm{j}, 1-\mathrm{j},-1+\mathrm{j},-1-\mathrm{j}$.

After going through SLM and RCF algorithm, the symbol sequence with the SI in the front is passed through the channel. At the receiver, the SI is first stripped off and stored for the latter use. The filtering is the inverse operation of the frequency domain filtering at the transmitter. That means, the sequence should be transformed by $\boldsymbol{N} \boldsymbol{L}$ points FFT, then its both ends are extracted to conduct the IFFT resulting in $\boldsymbol{R}-\boldsymbol{x}_{\boldsymbol{t}}$. The filtering process is shown as follows.

$$
\begin{aligned}
& f_{k}=\frac{1}{\sqrt{N L}} \sum_{i=0}^{N L-1} R-c_{i} \cdot e^{-j \frac{2 \pi}{N L} \cdot k i}, k=0,1, \cdots, N L-1 \\
& \left(R-x_{t}\right)_{k}= \begin{cases}f_{k}, & k=0,1, \cdots, \frac{N}{2}-1 \\
f_{k+N L-N}, & k=\frac{N}{2}, \cdots, N-1\end{cases}
\end{aligned}
$$

The $\boldsymbol{k}$ denotes the $\boldsymbol{k}$ th subcarrier, $\boldsymbol{f}_{\boldsymbol{k}}$ is the factor in the $\boldsymbol{k}$ th subcarrier after the FFT operation, and $\boldsymbol{R}-\boldsymbol{x}_{\boldsymbol{t}}$ is the sequence with $\boldsymbol{N}$ factors. $\boldsymbol{R}-\boldsymbol{x}_{\boldsymbol{t}}$ is corresponding to $\boldsymbol{x}_{\boldsymbol{t}}$ at the transmitter. The following two steps are the inverse operation of the SLM scheme. And then the sequence $\boldsymbol{R}-\boldsymbol{X}$, including $\boldsymbol{N}$ complex numbers, is obtained. After the de-mapping procedure, the output data stream $\boldsymbol{R}-\boldsymbol{x}$ is attained.

In summary, the proposed method combines SLM algorithm with RCF algorithm, which is a significant innovation in many occasions of reducing the PAPR.

\section{Simulations and Analysis}

\section{A. Simulation Condition}

The simulation conditions are given as follows. QPSK and 16QAM modulation, 128 and 256 subcarriers, 10000 OFDM symbol frames, and Additive Gaussian White Noise (AWGN) channel are adopted. QPSK modulation adopts Gray code mapping rules, which means the data stream $00,01,11,10$ is mapped to $-1-\mathrm{j},-1+\mathrm{j}, 1+\mathrm{j}, 1-\mathrm{j}$ respectively. The Signal-toNoise Ratio (SNR) varies between $0 \mathrm{~dB}$ and $20 \mathrm{~dB}$. The CR is equal to 4 corresponding to $6 \mathrm{~dB}$. As mentioned above, only the methods and steps of influencing PAPR are considered, and all other interferences except AWGN are ignored.

\section{B. Results and Discussion}

In this section, the simulation results of PAPR reduction and BER performance are presented and compared, which 
correspond to the proposed method, the RCF algorithm and the original OFDM system. As shown in Fig. 5.

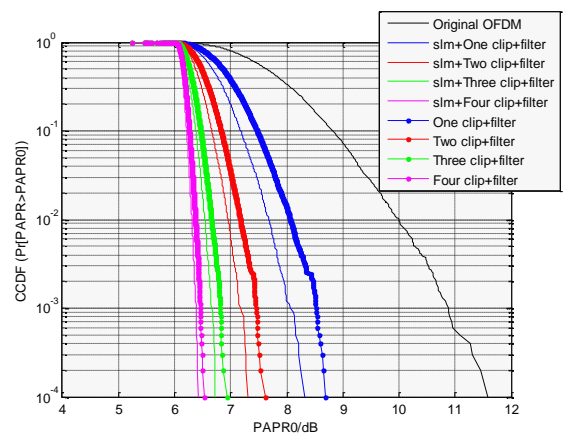

Fig. 5 CCDF of the PAPR for the Original OFDM System, the RCF Scheme and the Proposed Algorithm with N=128 and QPSK Modulation

In Fig. 5, the "one clip+filter" stands for a RCF operation. The CCDF of the PAPR is shown respectively in the original OFDM system, the only RCF applied system, and the joint scheme method applied system.

It can be seen that the PAPR of the traditional OFDM system is up to $11.5 \mathrm{~dB}$ when at the $0.01 \%$ CCDF probability level. The PAPR reduces to $8.7 \mathrm{~dB}$ and $8.3 \mathrm{~dB}$ while applying the RCF technique and the joint scheme respectively. Compared the PAPR of the RCF algorithm with the joint scheme, it can be observed that the proposed method is superior to the RCF algorithm clearly. Further, the PAPR reduction is about $0.4 \mathrm{~dB}$ and $0.3 \mathrm{~dB}$, and can up to $8.3 \mathrm{~dB}$ and $7.3 \mathrm{~dB}$ when RCF scheme is adopted once and twice respectively. In addition, when RCF scheme is adopted for three and four times, the PAPR of the proposed method can be dropped to $6.4 \mathrm{~dB}$ and $6.7 \mathrm{~dB}$.

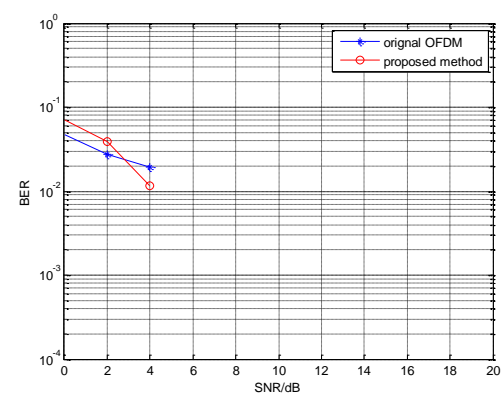

Fig. 6 The BER Performance of Original OFDM Systemand the Proposed Method with $\mathrm{N}=128$ and QPSK Modulation

In Fig. 6, the BER performances of the original OFDM system and the proposed method are shown. It can be seen that they can generate similar BER performance characteristics. When the SNR is equal to $0 \mathrm{~dB}$, it signifies that the signal power is equal to noise power. At this time, the BER is about $6 \%$ in the original OFDM system in Fig. 6, and the BER becomes $8 \%$ with the proposed method. It can be seen that the BER performance gets better when the number of the subcarriers is less. It demonstrates that the proposed method produces little distortion.

From the simulation results, it can be seen that much PAPR reduction is obtained than those in the system only with RCF scheme. And the BER performance is almost identical to that in the original OFDM system. So the method proposed in the paper proves to be very effective.

\section{Conclusion}

In this paper, a kind of method combining SLM with RCF algorithm for PAPR reduction is proposed. It makes full use of advantages of the two algorithms. There are prominent advantages in the proposed method. Firstly, compared with the PAPR in the original OFDM system, the PAPR reduction is evident. Secondly, the BER performance doesn't worsen and is even identical to that in the original OFDM system when different modulation schemes and different number of subcarriers are used. Furthermore, the proposed method is simple to implement and has no limitations on the system parameters such as the number of subcarriers, modulation mode, and so on.

In addition, in the past, OFDM was used rarely in WSN. With the progress of communication technology and digital circuit manufacture level, OFDM has gradually used in WSN either. So we must pay great attention to the application of OFDM in WSN.

\section{Acknowledgement}

The research group expresses heartfelt thanks to Professor Qin Tuanfa of Computer and Electronic Information College of Guangxi University, who has made great contributions to WSN. And he has put forward a lot of valuable opinions and suggestions for the work of research group.

The research has also got help from relating staffs of our unit. Hereon, we thank for them all together.

\section{References}

[1] Hangjun Chen and Alexander M. Haimovich, "Iterative Estimation and Cancellation of Clipping Noise for OFDM Signals," IEEE Communications Letters, vol. 7, no. 7, pp. 305-307, July 2003.

[2] Sulaiman A. Aburakhia, Ehab F.Badran, and Darwish A.E.Mohamed, "Linear Companding Transform for the Reduction of Peak-to-Average Power Ratio of OFDM Signals," IEEE Transactions on Broadcasting, vol.55, no.1, pp. 155-160, Mar. 2009.

[3] Athinarayanan Vallavaraj, Brian G Stewart, David K Harrison, Francis G McIntosh, "Reducing the PAPR of OFDM using a simplified scrambling slm technique with no explicit side information," IEEE computer society, doi 10.1109/ICPADS.2008.36. pp. 902-907, 2008.

[4] Armstrong, J. "New OFDM peak-to-average power reduction scheme," Proc.IEEE, VTC2001 Spring, Rhodes, Greece, 2001.

[5] Jean. Armstrong, "Peak-to-average power reduction for OFDM by repeated clipping and frequency domain filtering," Electronics Letters, vol. 38, no. 5, pp. 246-247, Feb. 2002.

[6] Ajit Jha, Md. Sohel Mahmud Sher, S.M.Shamsul Alam, Md, Tariq Hasan and Md. Mizanur Rahman, "Reduction of Peak to Average Ratio (PAPR) in Orthogonal Frequency Division Multiplexing(OFDM): a novel approach Based on clipping and amplification," IEEE Computer Society, pp. 921-926, 2009. 